\title{
O Grupo de Pesquisa Tecnologias no ensino e Atividades Experimentais: Possibilidades para potencializar os processos de ensino e de aprendizagem nas Ciências Exatas do Programa de Pós Graduação em Ensino de Ciências Exatas, Univates
}

The Research Group Technologies in Teaching and Experimental Activities: Possibilities to enhance lhe teaching and learning processes in the Exact Sciences of the Graduate Program in Exact Science Teaching, Univates

El Grupo de Investigación Tecnologías en la Docencia y Actividades Experimentales: Posibilidades de potenciar los procesos de enseñanza y aprendizaje en las Ciencias Exactas del Programa de Posgrado en Docencia de las Ciencias Exactas, Univates

Italo Gabriel Neide (italo.neide@univates.br) Universidade do Vale do Taquari - UNIVATES Maria Madalena Dullius (madalena@univates.br) Universidade do Vale do Taquari - UNIVATES

Marli Teresinha Quartieri (mtquartieri@univates.br) Universidade do Vale do Taquari - UNIVATES

Andréia Spessatto De Maman (andreiah2o@univates.br) Universidade do Vale do Taquari - UNIVATES

Ana Paula Krein Muller (anapmuller@universo.univates.br) Universidade do Vale do Taquari - UNIVATES

Geovana Luiza Kliemann (geovanakliemann@universo.univates.br) Universidade do Vale do Taquari - UNIVATES

Resumo: Neste trabalho é apresentado de forma geral o Programa de Pós Graduação em Ensino de Ciências Exatas (PPGECE) da Universidade do Vale do Taquari (Univates) localizada em Lajeado, Rio Grande do Sul, Brasil. Após a exposição da área de concentração e das linhas de pesquisa são apresentados os quatro macroprojetos que constituem os quatro grupos de pesquisa do PPGECE bem como sua descrição. Em seguida é dada ênfase a um grupo específico, o "Tecnologias no ensino e Atividades Experimentais: Possibilidades para potencializar os processos de ensino e de aprendizagem nas Ciências Exatas", apresentando seus aportes teóricos bem como as metodologias de pesquisa e de ensino utilizadas. Para compreender com propriedade como esse grupo realiza a construção do conhecimento científico apresenta-se de forma sintética três teses de doutorado que foram desenvolvidas pelo grupo. Ao final é Recebido em: 30/05/2021

Aceite em: 13/08/2021 
apresentado quantitativos gerais do grupo, bem como seu possível impacto na sociedade.

Palavras-chave: recursos tecnológicos; grupo de pesquisa; ensino de ciências.

Abstract: In this work, the Postgraduate Program in Exact Sciences Teaching (PPGECE) of the University of Vale do Taquari (Univates) located in Lajeado, Rio Grande do Sul, Brazil is presented. After exposing the concentration area and the lines of research, the four macro-projects that constitute the four research groups of the PPGECE are presented, as well as their description. Then, emphasis is given to a specific group, the "Technologies in Teaching and Experimental Activities: Possibilities to enhance the teaching and learning processes in the Exact Sciences", presenting its theoretical contributions as well as the research and teaching methodologies used. In order to properly understand how this group carries out the construction of scientific knowledge, three doctoral theses that were developed by the group are presented in a synthetic way. At the end, general figures for the group are presented, as well as its possible impact on society.

Keywords: technological resources; research group; science teaching.

Resumen: En este trabajo, se presenta el Programa de Posgrado en Docencia de Ciencias Exactas (PPGECE) de la Universidad de Vale do Taquari (Univates) ubicada en Lajeado, Rio Grande do Sul, Brasil. Tras exponer el área de concentración y las líneas de investigación, se presentan los cuatro macroproyectos que constituyen los cuatro grupos de investigación del PPGECE, así como su descripción. Luego, se hace énfasis en un grupo específico, el de "Tecnologías en la Docencia y Actividades Experimentales: Posibilidades de potenciar los procesos de enseñanza y aprendizaje en las Ciencias Exactas", presentando sus aportes teóricos así como las metodologías de investigación y docencia empleadas. Para comprender adecuadamente cómo este grupo lleva a cabo la construcción del conocimiento científico, se presentan de forma sintética tres tesis doctorales que fueron desarrolladas por el grupo. Al final se presentan las cifras generales del grupo, así como su posible impacto en la sociedad.

Palabras-clave: recursos tecnológicos; grupo de investigacion; enseñanza de las ciencias.

\section{INTRODUÇÃO}

Neste trabalho pretende-se apresentar os grupos de pesquisa do Programa de Pós Graduação em Ensino de Ciências Exatas (PPGECE) da Universidade do Vale do Taquari, Univates, localizada em Lajeado, Rio Grande do Sul. O PPGECE foi implantado em 2007, é de modalidade Profissional, e em 2019 aprovou o nível Doutorado. Sua finalidade é formar profissionais nas diversas áreas de ensino que busquem pensar a formação de professores, práticas pedagógicas inovadoras e novos

Recebido em: 30/05/2021

Aceite em: 13/08/2021 
olhares sobre o ensino. Nessa ótica, tem como missão a qualificação da formação profissional docente na área de Ensino de Ciências Exatas, em todos os níveis de ensino.

O Programa, por ser Profissional, prima pelas investigações serem efetivadas pelos discentes nas Instituições onde estes atuam, o que pode produzir significativos impactos na docência desses professores e de seus colegas. Assim, o foco está na pesquisa translacional e no desenvolvimento de Produtos Educacionais que sejam efetivamente implementados e avaliados em condições reais de ensino (podendo ser efetivada em espaços formais ou não formais). Para isso, são proporcionados momentos de vivência e reflexão constantes sobre a prática pedagógica imbricados com referenciais teóricos, discutindo-se e problematizando-se conteúdos, metodologias, avaliação, bem como questões sobre a própria formação docente. $\mathrm{O}$ intuito é qualificar profissionais que estão em exercício, visando melhorar os processos de ensino, de aprendizagem e de avaliação por meio da solução de problemas e geração e aplicação de processos de inovação apropriados, em todos os níveis de ensino na área das ciências exatas.

A área de concentração do programa é "Ensino de Ciências Exatas", com as seguintes linhas de pesquisa: Formação de professores, Processos de Avaliação para o Ensino de Ciências, Metodologias de Ensino e de Aprendizagem e Recursos Tecnológicos nos Processos de Ensino e de Aprendizagem. Para contemplar as linhas de pesquisa o PPGECE conta com 4 grupos de pesquisas norteados pelos macroprojetos descritos abaixo:

Ensinando, aprendendo e desenvolvendo produtos educacionais nas Ciências. Pretende problematizar como o ensino, norteado por metodologias ativas e participativas e por tecnologias digitais promove (potencializa) uma aprendizagem com significado na área das Ciências, nos diferentes níveis de ensino.

Práticas, ensino, currículo e formação docente no campo das ciências exatas. O macroprojeto compreende que práticas, ensino, currículos, avaliação e formação de professores são os eixos temáticos estruturantes que se articulam em uma rede que visa melhorar os processos de ensino e de aprendizagem em Ciências Exatas e diferentes níveis educacionais e contextos educativos.

Recebido em: 30/05/2021

Aceite em: 13/08/2021 


\section{Educação em Ciências: ensino e aprendizagem, articulações entre pesquisa e} práticas profissionais. O grupo tem como objetivo geral investigar como as práticas e saberes docentes de Ciências, podem contribuir para a formação dos estudantes.A proposta está embasada na pesquisa qualitativa, contemplada na pesquisa-ação, os dados coletados serão analisados, mediante Análise Textual Discursiva (ATD) ou Análise de Conteúdo.

\section{Tecnologias no ensino e Atividades Experimentais: Possibilidades para} potencializar os processos de ensino e de aprendizagem nas Ciências Exatas. $O$ objetivo deste macroprojeto é investigar o ensino e a aprendizagem de conteúdos das ciências exatas com a integração de tecnologias digitais e uso de atividades experimentais, analisar suas potencialidades e estruturar, explorar e discutir propostas de ensino.

Será dada ênfase para este último grupo de pesquisa apresentado. Ele é formado por cinco docentes permanentes do programa, doutorandos, mestrandos, bolsistas de iniciação científica e professores da rede privada e pública que são colaboradores. Teve início quando o PPGECE foi implementado, porém com outra formação e outros tópicos e vem a se consolidar definitivamente em 2013.

Dentre as ações desenvolvidas pelo grupo pode-se destacar: pesquisas científicas, atividades de extensão, formação|continuada de professores, desenvolvimento de produtos educacionais e livros, eventos destinados para alunos e professores, reuniões semanais e seminários científicos. Os principais aportes teóricos utilizados são "recursos tecnológicos", "atividades experimentais investigativas", "aprendizagem significativa", "metacognição", "modelagem científica" e "mentoring". No campo da metodologia de pesquisa utilizam-se da "Design Based Research", características de "Estudo de Caso" e "Pesquisa Ação", sendo que para análise de resultados utilizam-se as técnicas de "Análise Textual Descritiva" e "Método Descritivo". Vale ressaltar que o grupo mantém parceria internacional com dois outros grupos, um de Portugal e outro da Espanha, inclusive doutorandas já fizeram missões de estudo para esses grupos estrangeiros. Mais informações podem ser encontradas em <https://www.univates.br/ppgece/pesquisa/projetos-de-pesquisa/detalhes/303>. 
Alguns trabalhos que podem servir como exemplos relacionados a produção intelectual desse grupo de pesquisa são: "Percepções dos professores sobre o uso do software Modellus em uma experiência de modelagem" (NEIDE et al., 2019), "Modelagem Computacional num curso de formação continuada: percepções dos professores" (QUARTIERI et al., 2019) e "Aproximando a Matemática e a Física por meio de Recursos Tecnológicos: Ensino Médio" (QUARTIERI; DULLIUS, 2016). A seguir serão apresentadas três teses desenvolvidas pelo mesmo grupo de pesquisa apresentado para poder dar ênfase num grupo em específico e aprofundar quanto às características, metodologias, técnicas e embasamento teórico desse grupo.

\section{APRESENTAÇÃO DAS TRÊS TESES}

A seguir serão apresentadas três teses de doutorado que foram desenvolvidas no grupo de pesquisa. São apresentados os objetivos, a questão de pesquisa, uma breve passagem pelo referencial teórico, a metodologia e uma síntese dos resultados. Vale ressaltar que as três teses foram defendidas no último ano e ainda não constam na página do programa a versão definitiva, portanto ainda não possuem a respectiva indicação bibliográfica.

\section{Primeira Tese}

$\mathrm{Na}$ pesquisa de doutorado intitulada: "Mentoring com professores dos Anos Iniciais na metodologia de atividades experimentais investigativas e concepção sobre a natureza de ciência”, propôs-se identificar mudanças nas concepções e prática dos professores dos Anos Iniciais participantes do mentoring para o Ensino de Ciências, utilizando como principal estratégia, atividades experimentais Investigativas.

Este estudo foi norteado pela seguinte questão: Como e por que o mentoring e o desenvolvimento de atividades experimentais investigativas promovem mudanças na prática do professor dos Anos Iniciais? A partir da questão principal, emergiu outra questão cujas respostas foram investigadas no decorrer da pesquisa: Como ocorre para o professor o processo de mudança nas concepções sobre a natureza de ciência e na sua prática de ensino de Ciências?

Recebido em: 30/05/2021

Aceite em: 13/08/2021 
A fim de permitir maior integração ao contexto investigado, os pressupostos teóricos que norteiam esta pesquisa estão focados na análise e discussão de aspectos relacionados a concepção sobre a natureza de ciência, esta, amplamente utilizada na literatura, compreendida como uma construção humana que perpassa por mudanças, [...] caracterizada por uma permanente interação entre pensar, sentir e fazer" (MOREIRA; OSTERMAN, 1993). Nessa perspectiva, Pauletti (2018) considera a importância do conhecimento científico ser livre de rótulos, passível de refutações e inacabado. $\mathrm{Na}$ continuidade do referencial teórico, aborda-se à utilização das atividades experimentais para o ensino de Ciências nos Anos Iniciais, em especial as investigativas, estas, interpretadas por Pinho Alves (2000) como ações mediadas pelo docente, este que considera as hipóteses dos alunos, o trabalho em grupo e permite o diálogo entre professor, aluno e conhecimento, "ultrapassando a simples manipulação de materiais" (CARVALHO et al., 1998, p. 21). Ademais, propôs-se uma discussão de diferentes modelos de formação continuada, até definir o foco do estudo na metodologia de formação Mentoring, este que tem como base um agente mediador, o mentor, que auxilia outro professor, menos experiente, a aprender alguma coisa que sozinho teria mais dificuldade. Assim, a mentoria é "a ação de influenciar, aconselhar, ouvir, ajudar a clarificar ideias e a fazer escolhas, guiar”, (VERGARA, 2016, p. 109).

No contexto investigado, propôs-se inicialmente uma etapa preliminar de formação continuada a um grupo de 33 professores, durante o ano de 2018 . No decorrer dos 9 encontros presenciais, foram problematizadas atividades e discussões acerca da metodologia experimental investigativa, com intuito de conhecer o perfil, forma de trabalho e concepções do grupo de professores e dentre estes, elencar as mentorandas que permitiram maior aproximação ao contexto escolar e perceber por meio delas, aspectos aos quais pôde-se incidir.

Ao término da etapa preliminar, fez-se o convite às professoras Ana e à Maria, que participaram do mentoring em 2019. Neste percurso colaborativo de planejamentos e intervenções com cada mentoranda, foi possível investigar as contribuições da DBR (Design Based Research), para qualificar as ações do mentoring, avaliar as contribuições desta metodologia de formação para auxiliar os professores no desenvolvimento de atividades experimentais investigativas, além de buscar analisar o

Recebido em: 30/05/2021

Aceite em: 13/08/2021 
processo de mudança na concepção sobre a natureza de ciência e da prática no ensino de ciências.

Esta etapa da pesquisa, do tipo qualitativa, é pautada pela metodologia de pesquisa DBR. Corrobora-se com Santiago (2018, p. 18) que a DBR é "uma metodologia inovadora para se compreender como, quando e por que inovações educacionais e tecnológicas funcionam de modo prático, envolvendo contextos e os sujeitos deles participantes", para proposição dessa metodologia, a equipe de pesquisa contribuiu na projeção do redesenho de aspectos a serem potencializados com as mentorandas, através da mediação da mentora, que integrava ambos os contextos (escolas e universidade). Os dados emergentes da etapa preliminar, com o grupo de professores, são apresentados com excertos, de forma interpretativa cronológica. Quanto ao mentoring, os dados coletados, foram analisados e estão organizados em três ciclos conforme a DBR orienta: Ciclo de iniciação e cultivo; Ciclo de cultivo e separação e Ciclo de redefinição.

Quanto à etapa preliminar ficou evidente que o ensino de ciências não era prioridade, as atividades experimentais pouco integravam a prática do grupo de professores e neste viés, a Física e a Química eram algo distante desta realidade. Isto, associado principalmente à insegurança por esta área e ao desconhecimento da metodologia experimental investigativa. As concepções sobre a natureza de ciência do grupo de professores estavam distantes de uma concepção construtivista e mesmo depois de um período de formação, poucos avanços foram identificados. Essa experiência permitiu que a pesquisadora fosse para o mentoring com uma base de conhecimento desse contexto e realizar um trabalho colaborativo com as mentorandas.

No decorrer dos ciclos de vivência do mentoring, foi possível evidenciar a importância para as professoras receberem apoio para empreender mudanças, principalmente na estruturação do planejamento. O tempo prolongado da formação associado a múltiplas e contínuas vivências criaram um forte vínculo entre mentorandas e mentora, o que posteriormente resultou num esforço de criar estratégias cautelosas para prosseguirem de forma independente. Essa metodologia de formação mostrou-se eficaz para professores experientes e, aliada à DBR, auxiliou a redesenhar a formação.

Recebido em: 30/05/2021

Aceite em: 13/08/2021 
Ao término deste estudo, é possível concluir que a mudança perpassa por apoiar e auxiliar o professor em suas necessidades pontuais (superar medos e fortalecer sua autoconfiança), a partir de uma relação colaborativa de respeito. O melhor desempenho dos alunos também contribuiu à mudança, pois Ana e Maria viram melhorias na aprendizagem e perceberam que eram capazes de fazer diferente, isso as encorajou. Logo, o ensino por meio de atividades experimentais investigativas passou a fazer sentido e a variante nas ações contribuíram para mudança nas concepções sobre a natureza de ciência. As professoras perceberam que há uma aproximação entre o ensino de ciências e a natureza da ciência, sendo essa concepção mutável dependendo de como é ensinada. Quando segue o viés investigativo é uma construção humana e quando ensinada por transmissão, assume papel de memorização, estanque. Em síntese, o fazer reflexivo levou à melhor compreensão filosófica, consequentemente, a concepção perpassa pelas ações e vice-versa (ciclo entrelaçado). Finaliza-se com evidências de que houve microrrupturas de pensamento e de ação, sempre alicerçados em hábitos, crenças, cultura, materiais, entre outros elementos.

\section{Segunda Tese}

A pesquisa de doutorado intitulada: "Uso de recursos experimentais e computacionais para o desenvolvimento do pensamento metacognitivo no ensino de Física" teve como objetivo analisar indícios de pensamento metacognitivo de alunos de Engenharia, na disciplina de Física I, quando são desafiados a solucionar situaçõesproblema nas quais podem fazer uso de material experimental ou de simulação computacional.

O estudo teve como aporte teórico a escolha dos referenciais, em especial, os da metacognição, embasados nos trabalhos de Rosa (2011) e Rosa (2014), sendo estes alicerçados em Flavell (1971), Flavell (1976) e Brown (1978). Para estes a metacognição é o conhecimento que o sujeito tem sobre seu conhecimento e a capacidade de regulação dada aos processos executivos, somada ao controle e à orquestração desses mecanismos (ROSA, 2014). Como atividades experimentais, entende-se atividades que envolvem material concreto e manipulável para os alunos. As atividades experimentais são enriquecedoras para o aluno, uma vez que elas dão um 
verdadeiro sentido ao mundo abstrato e formal das linguagens. [...] ensinam as técnicas de investigação, possibilitam um olhar crítico sobre os resultados. [...] o aluno é preparado para poder tomar decisões na investigação e na discussão dos resultados (SERÉ; COELHO; NUNES, 2003, p. 39). Além disso, as atividades experimentais devem ser desafiantes, o aluno deve dedicar mais tempo refletindo do que manuseando equipamentos e vidrarias (GALIAZZI et al., 2001).

Já para as atividades virtuais, consideraram-se os ensinamentos de Araujo, Veit e Moreira (2008) quando apontam o uso da tecnologia como uma complementação às atividades experimentais, no sentido de que muitos experimentos são impossíveis de serem realizados em sala de aula, seja pela sua complexidade, perigo ou espaço físico. Corroborando com as ideias de Medeiros e Medeiros (2002) quando apontam as simulações com potencial de oferecer múltiplas representações simultaneamente de um determinado fenômeno físico (fórmula - tabela - gráfico - animação), fato que favorece a compreensão do fenômeno. As tecnologias também podem ser utilizadas como elemento de inclusão como pode ser evidenciado no trabalho "A importância das tecnologias numa perspectiva de Inclusão Digital para a prática docente" (SILVA; OLIVEIRA; COUTINHO, 2021), assim como elementos para formação de professores, que pode ser observado no trabalho "Formação inicial de professores de Ciências da Natureza: relatos de uma prática docente diferenciada" (COUTINHO; MIRANDA, 2019).

Assim, desenvolveu-se uma prática pedagógica, organizada em três intervenções didáticas, no Ensino Superior e na disciplina de Física I, composta de dezesseis alunos, na área de atuação da professora/pesquisadora, utilizando-se três roteiros-guia. A pesquisa é de cunho qualitativo e para a coleta de dados foram utilizados questionários, respondidos tanto de forma escrita como oral, e, posteriormente, transcritos nos diários de campo. A análise dos dados emergentes ocorreu por meio da elaboração de categorias a priori, usando características de Análise Textual Discursiva.

Após realizada a referida análise conclui-se que, mediante a utilização de recursos experimentais ou simulações computacionais, foi possível evocar o pensamento metacognitivo dos estudantes de Física de Cursos de Engenharia. Para que essa evocação acontecesse, o uso de roteiros-guia com questionamentos metacognitivos foi

Recebido em: 30/05/2021

Aceite em: 13/08/2021 
essencial para o diagnóstico deste resultado. As indagações estimularam os alunos a identificarem o que sabiam em relação ao tema de cada situação-problema e quais eram as melhores estratégias para se chegar à conclusão, além de se autorregularem ao longo do processo e avaliarem suas conclusões, desenvolvendo a sua autonomia e o senso crítico.

Com esta pesquisa, foi possível compreender a metacognição como uma aliada da promoção dos processos de ensino e de aprendizagem por meio de indícios do pensamento metacognitivo dos alunos participantes deste processo. Constatou-se também que, para a evocação do pensamento metacognitivo, é essencial que sejam promovidos espaços para que os alunos se autoconheçam, reflitam e pensem sobre seus conhecimentos como forma de aprimorar seu aprendizado. Além disso, observou-se que os elementos metacognitivos (pessoa, tarefa, estratégia, planificação, monitoração e avaliação) precisam atuar juntos, pois se influenciam mutuamente no sentido de auxiliar na ativação do pensamento metacognitivo.

Os sujeitos envolvidos na pesquisa, ao traçarem estratégias para a compreensão do conteúdo envolvido nas atividades dos roteiros-guia, com o auxílio de diferentes recursos, entre eles, das atividades experimentais e das simulações computacionais, apresentaram melhoras nos processos de aprendizagem e no conhecimento de conceitos da Física. Esse avanço metacognitivo foi significativo não só para a disciplina de Física I, mas por ser produtivo para outras disciplinas e a vida dos investigados. Em efeito, a escolha das melhores estratégias favorece a resolução de problemas de forma mais rápida e eficiente, otimizando o tempo e produzindo autonomia na busca de soluções.

Além disso, faz-se necessário destacar o papel da professora/pesquisadora neste processo. O professor pode desenvolver situações para promover o pensamento metacognitivo de seus estudantes, e sua função é ser mediador e agir como um promotor da autorregulação, ou seja, ensinar o aluno a planejar e monitorar suas atividades. Portanto, cabe a ele orientar e fomentar o pensamento metacognitivo dos seus discentes, estimulando-os a aprender a aprender e a aprender a pensar de forma autônoma e crítica, não só no âmbito da escola, universidade, mas como atitudes e estratégias para a vida.

\author{
Terceira Tese
}

Recebido em: 30/05/2021

Aceite em: 13/08/2021 
$\mathrm{Na}$ pesquisa de doutorado intitulada "Desenvolvimento profissional de professores dos anos iniciais na perspectiva de estudos de aulas por meio de recursos tecnológicos e atividades experimentais" apresenta-se uma investigação que buscou o desenvolvimento profissional de um grupo de professoras dos anos iniciais de uma escola pública de um município do interior do Rio Grande do Sul. O intuito foi fomentar práticas pedagógicas envolvendo a utilização de recursos tecnológicos e atividades experimentais para o ensino de ciências e matemática.

Esta pesquisa teve como objetivo geral analisar como a formação continuada baseada na metodologia de Estudos de Aula com professores dos Anos Iniciais e com foco em recursos tecnológicos e atividades experimentais, pode promover $\mathrm{o}$ desenvolvimento profissional desses docentes. E como objetivos específicos buscou-se: constatar as conjecturas dos professores em relação a utilização de recursos tecnológicos e o desenvolvimento de atividades experimentais com alunos dos Anos Iniciais; identificar implicações dos professores dos Anos Iniciais no decorrer de momentos de formação continuada em contextos colaborativos, em relação à utilização dos recursos tecnológicos e das atividades experimentais em suas práticas pedagógicas; investigar potencialidades da metodologia de estudo de aula no desenvolvimento profissional de um grupo de professores dos Anos Iniciais; e investigar as contribuições da DBR para aperfeiçoar os encaminhamentos da metodologia de Estudos de Aula durante o processo de formação continuada.

Para os Estudos de Aula foram utilizadas as ideias de autores como: Merichelli e Souza (2016) que consideram uma proposta eficaz de formação de professores num curso de formação continuada. Curi (2018, p. 19) também destaca que "é um processo de desenvolvimento profissional de professores, organizados em grupos colaborativos, mediados por pesquisadores, a partir da tematização da prática de sala de aula!”. As autoras condensam a metodologia Estudos de Aula em três etapas fundamentais para o processo de desenvolvimento, sendo que a primeira refere-se ao planejamento das aulas, realizada em grupos colaborativos formados por professores e pesquisadores. A segunda tem o foco no desenvolvimento das atividades de ensino planejadas por um dos professores participantes, com os demais fazendo o papel de observadores do processo, e uma terceira etapa, quando os professores e pesquisadores analisam e discutem as observações e as falas dos envolvidos.

Recebido em: 30/05/2021

Aceite em: 13/08/2021 
Em relação ao desenvolvimento profissional do professor utilizou-se bases teóricas de autores como Imbernón (2011), que aponta que a formação continuada é considerada um dos elementos para promover o desenvolvimento profissional do docente. E corroborando com o processo de formação Ponte (1998) destaca que o mais importante é que o professor deixe de ser um profissional que somente recebe a formação, e passe a ser sujeito da formação.

A investigação perpassou três etapas de investigação, sendo que em 2017, realizou-se um grupo focal para identificar e compreender as principais angústias, dificuldades e necessidades desse grupo de professoras. A partir desses dados, organizou-se um curso de formação no ano de 2018, nos moldes da constituição de grupos colaborativos e distribuídos em 9 encontros, dos quais participaram 11 professoras. Nestes momentos as professoras tiveram a oportunidade de compartilhar ideias, sugestões e práticas pedagógicas já desenvolvidas, além de discutir questões teóricas envolvendo a utilização de recursos tecnológicos e o desenvolvimento de atividades experimentais para o ensino de Ciências e Matemática dos Anos Iniciais.

Já em 2019, foram organizados momentos de encontros com dois grupos de professoras (A e B), no qual foi utilizada a metodologia de Estudos de Aula para fomentar o desenvolvimento e a continuidade do processo de formação. Neste período de Estudos de Aula, foram desenvolvidos 4 ciclos completos com cada um dos dois grupos, sendo que os ciclos envolveram o planejamento, aplicação e observação, avaliação e replanejamento e avaliação final de uma prática pedagógica com as professoras participantes. Os conteúdos desenvolvidos com os alunos em cada ciclo foram escolhidos pelas professoras de cada grupo, considerando suas turmas.

A pesquisa teve como base um estudo qualitativo fundamentado na metodologia de Design Based Research (DBR). Os dados coletados foram por meio de gravações de áudio, filmagem, diário de campo da pesquisadora e diário de anotações das participantes. A análise de dados da pesquisa foi organizada de forma cronológica, sendo que na realização do grupo focal, as participantes destacaram dificuldades em relação a disponibilidade de tempo para planejamento e preparação de atividades envolvendo a utilização destes recursos, a falta de hábito e a insegurança com os equipamentos e os conceitos teóricos envolvidos. Nos encontros de 2018, percebeu-se 
que as professoras se envolveram na realização das atividades experimentais, participando das discussões e realizando apontamentos e considerações de como essa atividade pode ser importante para abordar alguns conteúdos em sala de aula. De forma geral percebeu-se que as professoras possuem pouco contato com materiais diferenciados. E, quando foram desafiadas a realizar a atividade sem a orientação da pesquisadora, ou seja, seguindo o roteiro fornecido, muitos sentimentos foram apresentados, como ansiedade, nervosismo, preocupação. No final, ocorreu empolgação e alegria, pois conseguiram realizar a experiência com sucesso, observando os resultados esperados. As professoras perceberam que atividades simples podem ser utilizadas para abordar os temas de Ciências e instigar os alunos a investigar e pesquisar diferentes questões.

Nos encontros, em 2019, em que foi utilizada a metodologia de Estudo de Aula, durante os momentos de planejamento percebeu-se o envolvimento e o entusiasmo das professoras, todas realizavam contribuições para organizar uma atividade que atendesse aos objetivos pensados, bem como se preocuparam com a aprendizagem dos seus alunos. A importância do processo de observação foi devido à aprendizagem que ocorreu entre colegas, a confiança que foi surgindo, no qual a professora se sentia à vontade para dar sugestões e críticas em relação a outra colega. Nos momentos de avaliação e replanejamento, todas contribuíram com observações realizadas e consideraram a metodologia de encontro com os pares produtiva para aperfeiçoamento das práticas pedagógicas. E para finalizar, a colaboração passou a ser percebida para além dos momentos de encontro dos Estudos de aula, quando as professoras se reuniam para trocar ideias e planejamentos. Neste sentido, destaca-se que os ciclos de Estudo de Aula proporcionaram momentos diferenciados para as participantes e de forma geral todas consideram os momentos de planejamento, observação e avaliação, importantes para o processo de formação e melhoria das práticas pedagógicas.

\section{CONSIDERAÇÕES FINAIS}

Neste artigo foram apresentados os grupos de pesquisa do PPGECE, e em ênfase o grupo "Tecnologias no ensino e Atividades Experimentais: Possibilidades para potencializar os processos de ensino e de aprendizagem nas Ciências Exatas”. Para tanto

Recebido em: 30/05/2021

Aceite em: 13/08/2021 
foram socializados os principais elementos teóricos do grupo, bem como seus sujeitos, suas ações e algumas de suas publicações intelectuais, destacando especificamente três teses de doutorado desenvolvidas no grupo de pesquisa. Elemento consoante nestes trabalhos é que objetivam a sala de aula, de forma que sempre manteve no horizonte a procura de uma transformação da prática docente para poder melhorar os processos de ensino e de aprendizagem. Os principais fatores que emergem dos resultados desses trabalhos é a autonomia dos sujeitos envolvidos.

$\mathrm{Na}$ primeira tese destaca-se a confiança dos professores com a formadora durante a formação, e a ruptura para que pudessem realizar os desenvolvimentos sozinhos. Na segunda tese foi socializado que é muito importante o planejamento com dedicação, com embasamento teórico, considerando sempre os objetivos da investigação. Já na terceira tese foi destacada a importância no trabalho colaborativo de docentes e a potencialidade desta interação colaborativa entre docentes.

Portanto pode-se notar que o grupo de pesquisa já conta com anos de experiência, produz frequentemente conhecimento científico e socializa seu conhecimento por meio de publicações em artigos científicos, publicações de livros, dissertações de mestrado e teses de doutorado. Tem parcerias internacionais e conta com um impacto social importante em várias instituições de ensino, independente do nível e do tipo da rede, seja ela pública ou privada.

\section{REFERÊNCIAS}

ARAUJO, I. S.; VEIT, E. A.; MOREIRA, M. A. Physics students' performance using computational modelling activities to improve kinematics graphs interpretation.

Computers \& Education, v. 50, p. 1128-1140, 2008. Disponível em: https://www.learntechlib.org/p/67185/. Acesso em: 5 jan. 2019.

BROWN, A. L. Knowing when, where, and how to remember: a problem of metacognition. In: GLASER, R. (ed.). Advances in instructional psychology. Hillsdale, New Jersey: Erlbaum, 1978. v. 1, p. 77-165. Disponível em:

https://files.eric.ed.gov/fulltext/ED146562.pdf. Acesso em: 3 jan. 2019.

CARVALHO, A. M. P de; VANNUCCHI, A. I; BARROS, M. A.; GONÇALVES, M. E. R.; REY, R. C. de. Ciências no Ensino Fundamental: O conhecimento físico. 1. ed. São Paulo: Scipione, 1998. 
COUTINHO, C.; MIRANDA, A. C. G. Formação inicial de professores de Ciências da Natureza: relatos de uma prática docente diferenciada. Revista Insignare Scientia-RIS, v. 2, n. 2, p. 221-231, 2019.

CURI, E. Reflexões sobre um projeto de pesquisa que envolve grupos colaborativos e a metodologia lesson study. In.: CURI, E.; NASCIMENTO, J. de C. P. do; VECE, J. P. (orgs). Grupos colaborativos e lesson study: contribuições para a melhoria do ensino de matemática e desenvolvimento profissional de professores. Alexa Cultural: São Paulo, 2018.

DA SILVA, E.; DE OLIVEIRA, A. J.; COUTINHO, D. J. G. A importância das tecnologias numa perspectiva de Inclusão Digital para a prática docente. Revista Insignare Scientia-RIS, v. 4, n. 1, p. 63-77, 2021.

FLAVELL, J. H. First discussant's comments: what is memory development the development of? Human Development, New York, EUA, n. 14, p. 272-278, 1971. Disponível em: https://www.jstor.org/stable/26761846?seq=1\#metadata _info_tab_contents. Acesso em: 10 abr. 2021.

FLAVELL, J. H. Metacognitive aspects of problem solving. In: RESNICK, L. B. (ed.). The nature of intelligence. Hillsdale, New Jersey: Erlbaum, 1976. p. 231-236.

GALIAZZI, M. C. et al. Objetivos das atividades experimentais no Ensino Médio: a pesquisa coletiva como modo de formação de professores de Ciências. Ciência \& Educação, Bauru, SP, v. 7, n. 2, p. 249-263, 2001. Disponível em: https://www.scielo.br/pdf/ciedu/v7n2/08.pdf. Acesso em: 10 abr. 2021.

IMBERNÓN, F. Formação Docente e Profissional: formar-se para a mudança e a incerteza. [tradução Silvana Cobucci Leite]. Coleção questões da nossa época; v. 14, ed. 9. São Paulo: Cortez, 2011.

MEDEIROS, A.; MEDEIROS, C. F. Possibilidades e limitações das simulações computacionais no ensino de Física. Revista Brasileira de Ensino de Física, São Paulo, v. 24, n. 2, p.77-86, 2002. Disponível em: http://www.scielo.br/pdf/rbef/v24n2/ a02v24n2.pdf. Acesso em: 22 out. 2018.

MOREIRA, M.A.; OSTERMANN, F. Sobre o ensino do método científico. Cad.Cat.Ens.Fís., v.10, n.2: p.108-117, ago. 1993.

MERICHELLI, M, A. J.; SOUZA, I. C. P. de. As aprendizagens profissionais de um grupo de professores em um estudo de aula. In: XII Encontro Nacional de Educação Matemática. Anais... São Paulo. Disponível em:

<http://www.sbembrasil.org.br/enem2016/anais/pdf/4723 3790_ID.pdf >. Acesso em: 04 out. 2018.

NEIDE, I. G. et al. Percepções dos professores sobre o uso do software Modellus em uma experiência de modelagem. Caderno Brasileiro de Ensino de Física, v. 36, n. 2, p. 567-588, 2019.

PAULETTI, F. A pesquisa como princípio educativo no ensino de ciências: concepções e práticas em contextos brasileiros. 2018. Tese de Doutorado em educação em ciências e matemática. PUCRS, 2018.

Recebido em: 30/05/2021

Aceite em: 13/08/2021 
PINHO-ALVES, J. Atividades experimentais: do método à prática construtivista. 2000. Tese Doutorado em Educação) - Universidade Federal de Santa Catarina, Florianópolis, 2000.

PONTE, J. P. Didácticas Específicas e Construção do Conhecimento Profissional. In. Investigar e formar em educação. Actas do IV Congresso da SPCE. Porto: Sociedade Portuguesa de Ciências da Educação. 1998. Disponível em:

<http://www.educ.fc.ul.pt/docentes/jponte/docs-pt/99-Ponte(Aveiro).pdf>. Acesso em: 03 jul. 2018.

QUARTIERI, M. T. et al. Modelagem Computacional num curso de formação continuada: percepções dos professores. Revista de Ensino de Ciências e Matemática, v. 10, n. 6, p. 274-286, 2019.

QUARTIERI, M. T., DULliUS, M. M. Aproximando a Matemática e a Física por meio de Recursos Tecnológicos: Ensino Médio. 1. ed. Lajeado: Editora Univates, 2016.

ROSA, C. T. W. DA. A metacognição e as atividades experimentais no ensino de Física. 2011. Tese (Doutorado em Educação Científica e Tecnológica) - Universidade Federal de Santa Catarina, Florianópolis, SC, 2011. Disponível em: https://repositorio.ufsc.br/xmlui/handle/123456789/95261. Acesso em: 15 jun. 2018.

ROSA, C. T. W. DA. Metacognição no ensino de Física: da concepção à aplicação. Passo Fundo, RS: UPF, 2014. E-book. Disponível em: http://editora.upf.br/ images/ebook/metacognicao_ensino_fisica.pdf. Acesso em: 8 abr. 2019.

SANTIAGO, R. C. C. de A. Framework design-based research para pesquisas aplicadas. 2018. $300 \mathrm{f}$. Tese (Doutorado multi-institucional e Multidisciplinar em Difusão do Conhecimento), Universidade Federal da Bahia. Faculdade de Educação, Salvador, 13 mar. 2018.

SÉRÉ, M. G.; COELHO, S. M.; NUNES, A. D. O papel da experimentação no ensino de Física. Caderno Brasileiro de Ensino de Física, Florianópolis, SC, v. 20, n. 1, p. 31 42, jan. 2003. Disponível em: https://periodicos.ufsc.br/index.php/fisica/ article/view/6560. Acesso em: 18 jan. 2019.

VERGARA, S. C. Gestão de pessoas. 16. ed. São Paulo: Atlas, 2016. 